\title{
Robot-Assisted Thoracoscopic Esophagectomy with Total Mediastinal Lymphadenectomy: A Guide to a Systematic Approach Using the Concept of Fascial Plane Dissection
}

\author{
Byung Jo Park, M.D., Dae Joon Kim, M.D., Ph.D. \\ Department of Thoracic and Cardiovascular Surgery, Yonsei University College of Medicine, Seoul, Korea
}

ARTICLE INFO

Received June 14, 2021

Accepted July 2, 2021

Corresponding author

Dae Joon Kim

Tel $82-2-2228-2140$

Fax 82-2-2228-2140

E-mail kdjcool@yuhs.ac

ORCID

https://orcid.org/0000-0002-2182-010X

\begin{abstract}
Recent case series and meta-analyses have suggested that robot-assisted minimally invasive esophagectomy (RAMIE) could be a useful alternative to video-assisted thoracic surgery esophagectomy. The advantages of RAMIE are a 3-dimensional view, 7 degrees of freedom, and tremor filtering, which enable more meticulous lymph node dissection with a lower incidence of complications. However, in radical esophagectomy, understanding the concepts of the fascia and compartment is crucial for successful and reliable dissection. The first RAMIE in Korea was performed by our team in July 2006, and since then, we have developed related techniques to achieve better short- and long-term outcomes. The key step in RAMIE for esophageal squamous cell carcinoma is dissection of the upper mediastinum due to the difficulty of lymph node dissection and the high incidence of nodal metastasis in the area. Herein, we describe the technique of fascial plane dissection with esophageal suspension during RAMIE.
\end{abstract}

Keywords: Esophagectomy, Robot-assisted, Lymph node dissection

\section{Introduction}

Since robot-assisted minimally invasive esophagectomy (RAMIE) was introduced in the mid-2000s, numerous case series and meta-analyses have suggested that it could be a useful alternative to video-assisted thoracic surgery esophagectomy (VATS-E). The currently used da Vinci Surgical System provides key functions such as 3-dimensional vision, tremor filtering, motion scaling, and the endo-wrist function, which are expected to decrease the incidence of morbidity after RAMIE compared to that after VATS-E [14]. Since the first RAMIE in Korea, performed by our team (Severance Hospital) in July 2006, we have developed useful techniques for radical esophagectomy with favorable postoperative and oncologic outcomes [5-9]. Although novel devices and technologies have been introduced in the past decade, understanding the microanatomy of the esophagus and related structures is a prerequisite for the success of RAMIE. Here, we present in detail a systematic approach to RAMIE using fascial plane dissection.

\section{Port placement and patient position}

After general endotracheal anesthesia with a bronchial blocker, the patient is turned to the semi-prone position (Fig. 1). An 8-mm port (P2) is placed at the sixth intercostal space (ICS), just below the inferior scapula tip, and a $30^{\circ}$ thoracoscope is inserted. After an assessment for pleural seeding or adhesion, $\mathrm{CO}_{2}$ gas is insufflated at a pressure of $8-10 \mathrm{~mm} \mathrm{Hg}$. An 8-mm port (P1) is placed at the third or fourth ICS on the posterior axillary line (PAL). In the case illustrated here, the target area was the thoracic inlet; thus, a mark was drawn on a straight line from the camera port towards it. Then, an $8-\mathrm{mm}$ port (P3) was inserted at the eighth or ninth ICS on the PAL, and another $8-\mathrm{mm}$ port (P4) was inserted at the 11th ICS on the scapular tip line. A 12-mm accessory port (PA) was inserted at the fifth or sixth ICS on the anterior axillary line. The robotic cart (da Vinci Xi) was positioned at the patient's left side. A green target from a laser-guided system was aligned with $\mathrm{P} 2$, and the camera was inserted through P2, targeted at the thoracic inlet. The cart then automatically adjusted its 


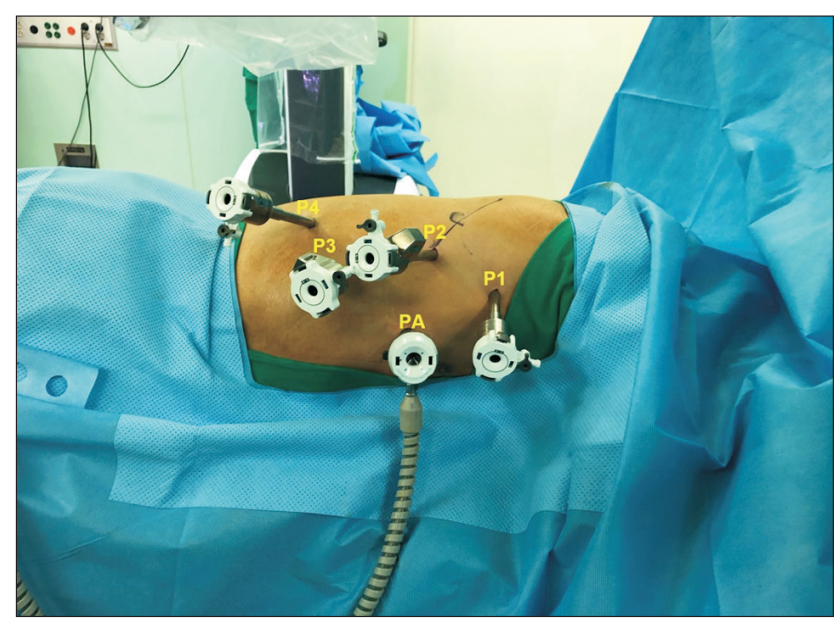

Fig. 1. Port placement and patient position for robot-assisted thoracoscopic esophagectomy: P1-P4 (8-mm port) and PA (12-mm accessory port).

boom in the optimized configuration, the remaining robotic arms were docked, and the rest of the instruments were inserted.

\section{Dissection of the upper mediastinum}

\section{Practical anatomy of the upper mediastinum}

Fig. 2 shows a schematic diagram of the upper mediastinum, superior to the aortic arch. The visceral compartment contains the esophagus and trachea, while the vascular compartment contains the major arteries and veins. Each compartment is surrounded by thin connective tissue layers (the visceral and vascular fascia). The vagus nerve descends along the carotid artery in the vascular compartment. The recurrent laryngeal nerve (RLN) branches from the vagus nerve penetrate the visceral fascia and run upward along the trachea in the visceral compartment. The cardiac branch of the sympathetic nerve is in the vascular compartment, and the thoracic duct travels between the visceral and vascular fascia. To clear all the lymph nodes around the RLN without nerve injury, attention should be focused on the relationship between the visceral fascia and the RLN at the left side, as most cases of RLN palsy occur in this area.

There are 2 major routes of lymphatic metastasis in esophageal cancer [10-13]. Intramural metastasis mainly occurs through the longitudinal lymphatic channels in the submucosal layer, whereas extramural metastasis occurs through peri-esophageal lymphatics. Thus, it is essential to

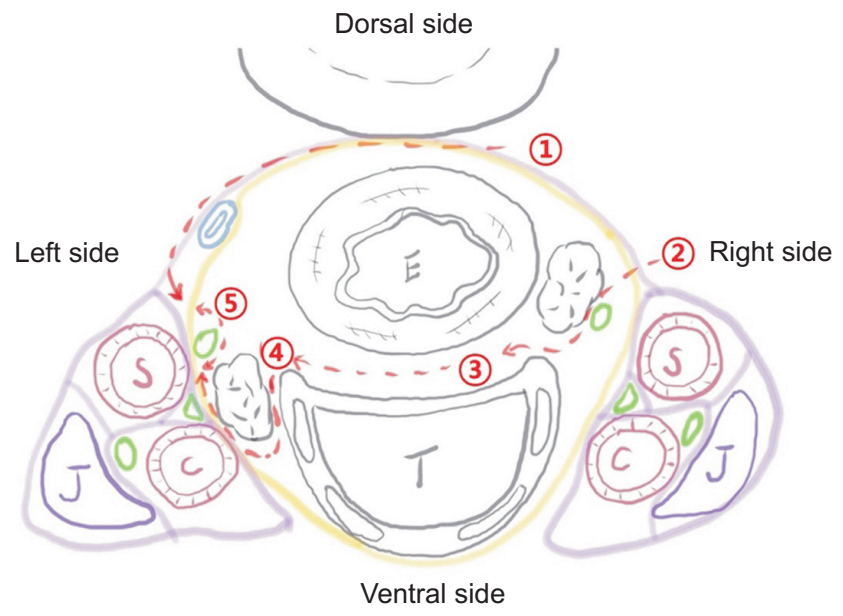

Fig. 2. Schematic drawing of the upper mediastinum superior to the aortic arch. Yellow line, vascular fascia; pink line, vascular fascia; fluorescent green circle, nerve; blue double circle, thoracic duct; E, esophagus; T, trachea; J, jugular vein; S, subclavian artery; C, common carotid artery; numbers and the red dotted line, dissection order.

dissect all lymphatic tissues in the visceral compartment to achieve excellent locoregional control. Another route of metastasis is the thoracic duct and its surrounding nodes. The thoracic duct receives lymphatic flow from abdominal lymph nodes and direct drainage from the esophagus with a nodal relay. This suggests the possibility of rapid lymph drainage into the systemic circulation via the thoracic duct in esophageal cancer. Although the effect of thoracic duct resection has yet to be established, we routinely dissect the thoracic duct en bloc with the esophagus.

\section{Dorsal part dissection}

A ProGrasp forceps is inserted through P3 to compress the lung while pulling the esophagus down. Dissection is begun with a curved bipolar dissector (through P4) and monopolar curved scissors (through P1). The azygos vein is cut after applying Hem-o-lok clips (Fig. 3A); then, the right bronchial artery is identified and cut with a vessel sealer (Fig. 3B). For routine resection of the thoracic duct, identification of the duct below the azygos vein is the next step (Fig. 3C). The dorsal side of the mediastinal pleura is opened (Fig. 3D). The thoracic duct is resected from its cranial part (Fig. 3E) to the most caudal part, and the caudal end is cut after clipping to prevent chylothorax (Fig. $3 \mathrm{~F})$. The dorsal side of the esophagus is subsequently dissected with monopolar curved scissors, and the dorsolateral part of the esophagus is mobilized. The left subclavian 

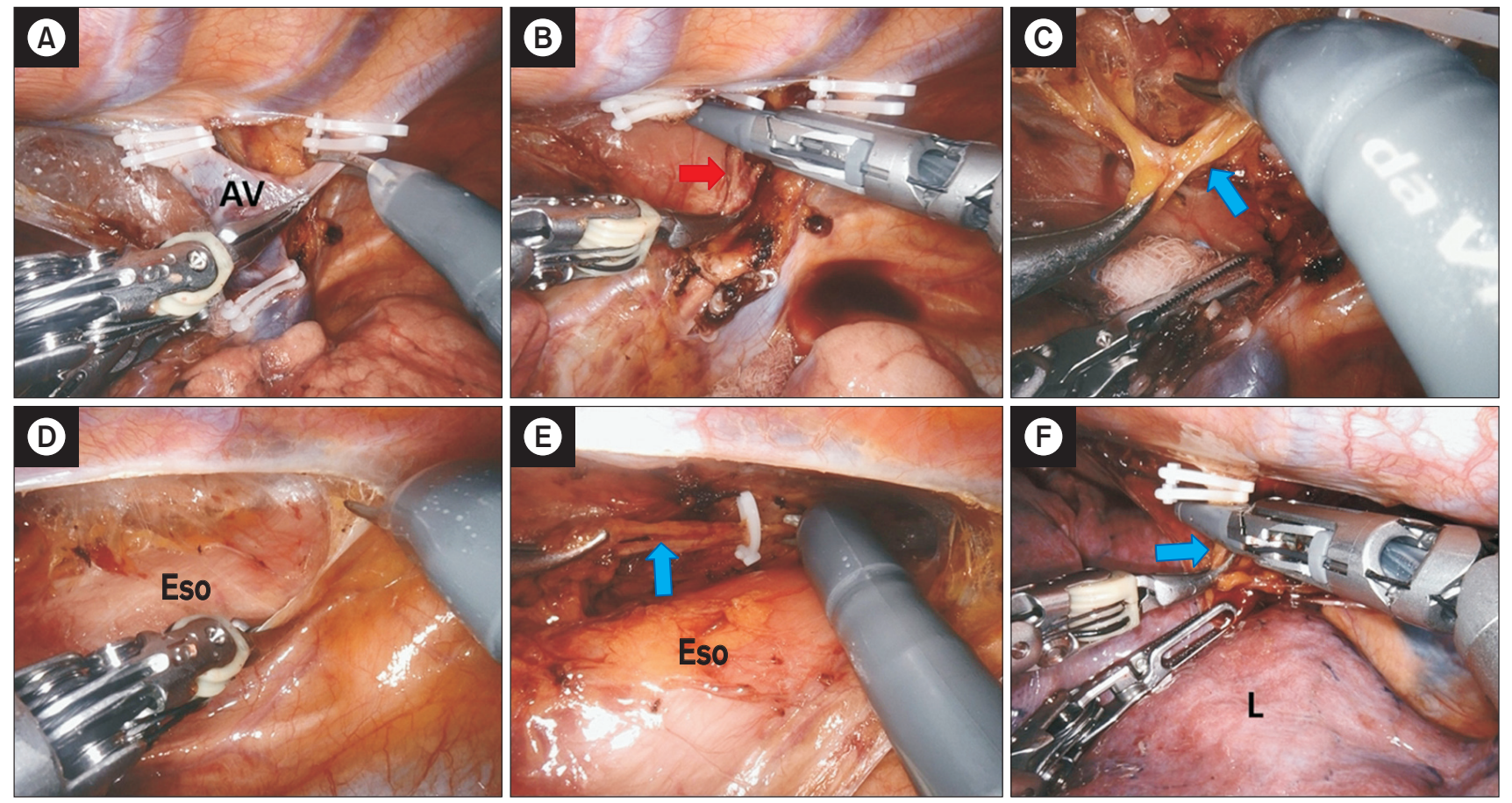

Fig. 3. Dissection of the dorsal part of the upper mediastinum. The thoracic duct is identified and resected after clipping. (A) Azygos vein (AV) division after applying Hem-o-lok clips. (B) Right bronchial artery division with vessel sealer extension. (C) Identification of the thoracic duct. (D) Dissection of the dorsal mediastinal pleura. (E, F) Resection of the thoracic duct. Eso, esophagus; L, lung; red arrow, right bronchial artery; blue arrow, thoracic duct.

artery need not be identified, as it is located outside the visceral compartment and its dissection with the energy device may cause thermal injury to the left RLN.

\section{Ventral part dissection}

A ProGrasp forceps is inserted through P4 to lift the esophagus, and a curved bipolar dissector is moved to P3. After lifting the esophagus, the right vagus nerve is identified, and the mediastinal pleura is opened toward the level of the subclavian artery (Fig. 4A). The vascular sheath over the right vagus nerve is opened, which allows identification of the right RLN, as it penetrates the visceral compartment in most patients (Fig. 4B). The visceral sheath over the right RLN is cut using curved scissors, along with the identified esophageal branches of the RLN (Fig. 4C). The lymphoadipose tissue posterior to the RLN is then dissected carefully.

The next step is to mobilize the esophagus from the trachea. The esophagus is lifted using a ProGrasp forceps (through P4), and the trachea is retracted to the right side with a grasper or a peanut dissector by an assistant (through PA) (Fig. 4D). The loose connective tissue between the esophageal muscle and the trachealis muscle is dissected, with careful avoidance of airway injury in the process. After the esophagus is completely mobilized from the trachea, the lymphoadipose tissue along the left lateral border of the trachea is dissected, and small vessels from the tracheoesophageal artery are cauterized with monopolar curved scissors. The visceral fascia, a shiny, glossy membrane-like structure that is the boundary of lymph node dissection in the upper mediastinum, can be identified underneath the lymphoadipose tissue.

\section{Dissection along the left recurrent laryngeal nerve}

After identification of the visceral fascia, a single strand of nylon tape is applied to encircle the esophagus and tie it at 3 points (Figs. 5A,6A). This maneuver, termed esophageal suspension, provides a wider operating space in the left paratracheal space while preventing nerve injury from excessive stretching or kinking during the procedure. Identification of the left RLN at the level of the tracheobronchial angle is the next step. After identification of the visceral fascia layer, the nerve can easily be identified as it is located 

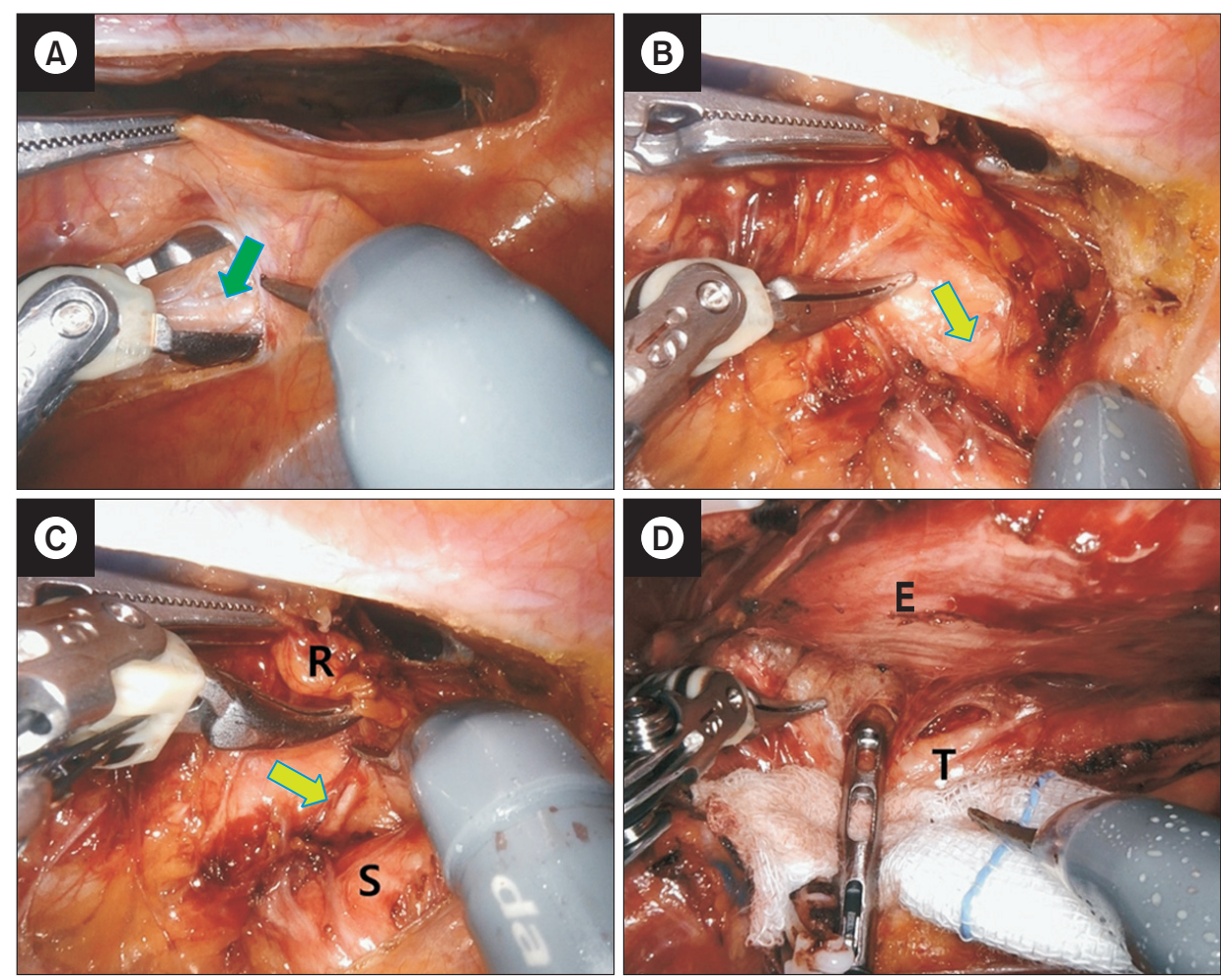

Fig. 4. Dissection of the ventral part of the upper mediastinum. Lymph nodes along the right recurrent laryngeal nerve (RLN) are dissected. (A) The mediastinal pleura above the vagus nerve is opened. (B) Identification of the right RLN penetrating into the visceral compartment. (C) Identification and incision of the esophageal branches of the RLN. (D) Mobilization of the esophagus from the trachea. R, right RLN lymph nodes; S, subclavian artery; $\mathrm{E}$, esophagus; $\mathrm{T}$, trachea; green arrow, vagus nerve; fluorescent green arrow, right RLN.
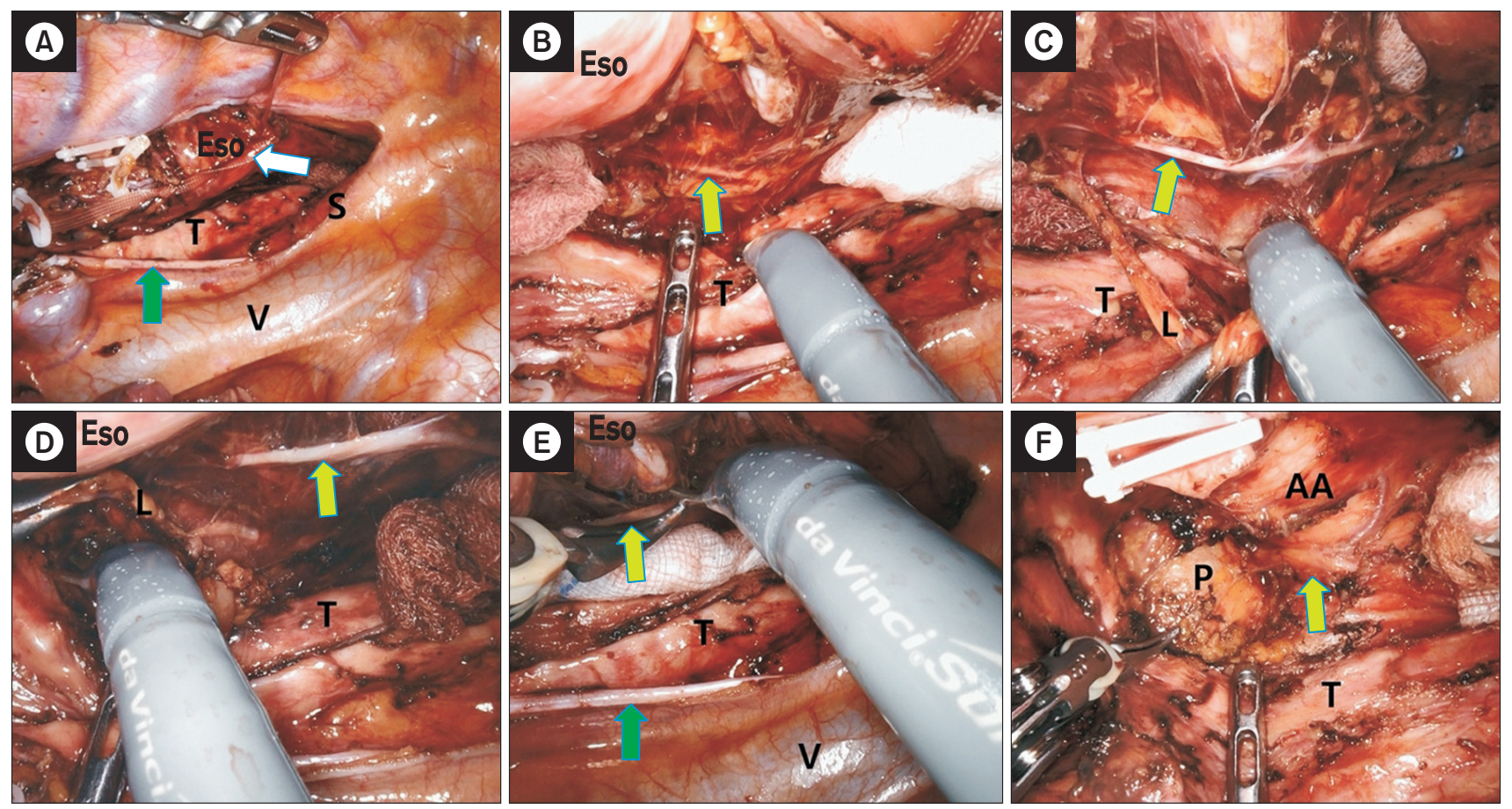

Fig. 5. Dissection along the left recurrent laryngeal nerve (RLN). (A) The esophagus is tied at 3 points using a single strand of nylon tape. (B) The left RLN is identified. (C, D) Left RLN lymph nodes are dissected. (E) The esophageal branches of the left RLN are cut. (F) Lymph nodes at the tracheobronchial angle and the infra-aortic area are dissected. Eso, esophagus; $\mathrm{T}$, trachea; $\mathrm{S}$, subclavian artery; $\mathrm{V}$, superior vena cava; L, left RLN lymph nodes; AA, aortic arch; P, left pulmonary artery; white arrow, nylon tape; green arrow, vagus nerve; fluorescent green arrow, left RLN. 

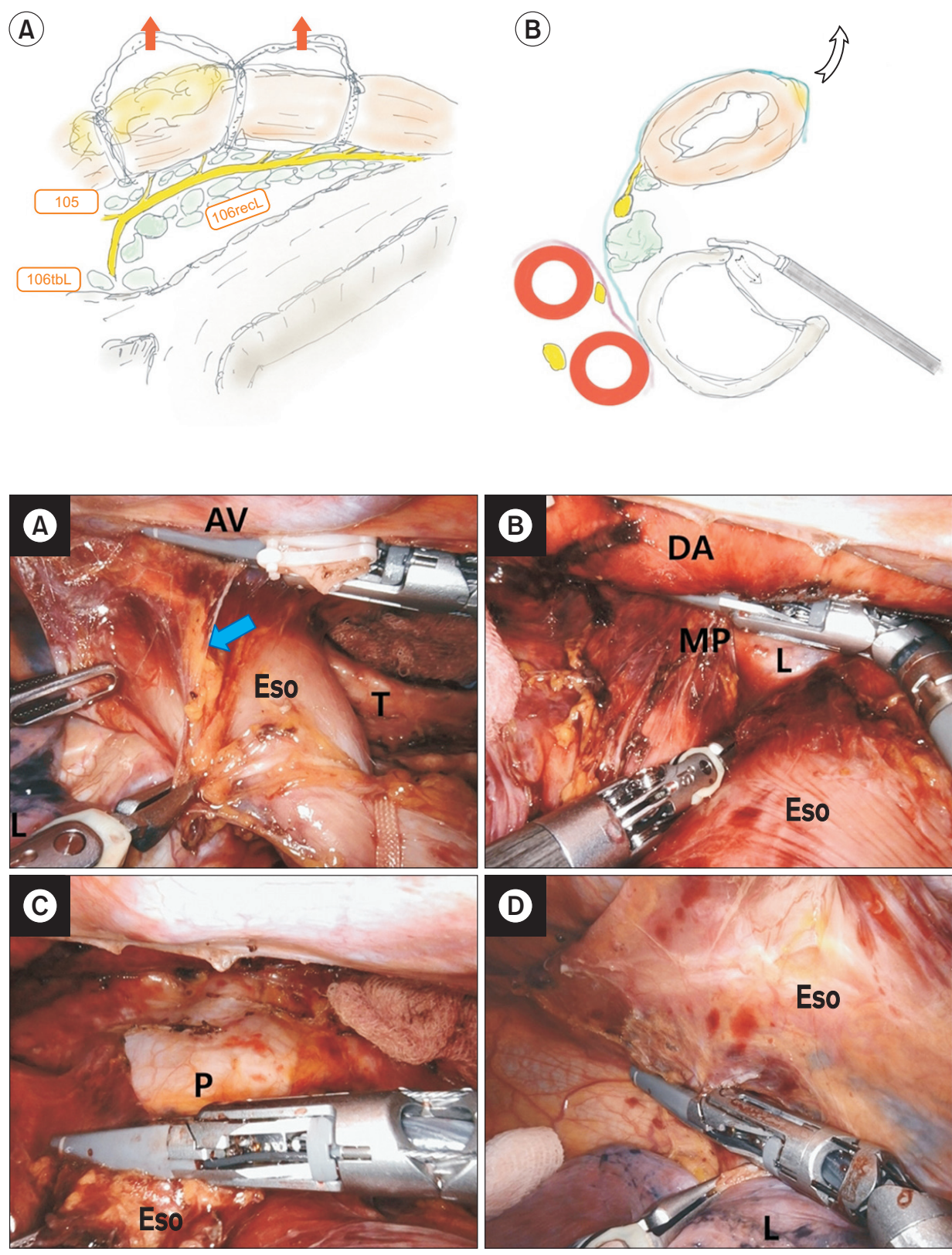

Fig. 6. Schematic drawing of dissection along the left recurrent laryngeal nerve (RLN). (A) Lateral view of the left RLN and lymph node station after the esophagus is retracted to the right side. (B) Transection view of the left RLN and lymph node station after the esophagus is retracted to the right side.
Fig. 7. Middle to lower mediastinum dissection. (A) Dissection of the thoracic duct. (B) Dissection of the dorsal side of the esophagus. (C) Dissection of the esophagus from the pericardium. (D) Dissection of right mediastinal pleural and lymphoadipose tissue of the right pulmonary ligament. $\mathrm{AV}$, azygos vein; Eso, esophagus; $T$, trachea; $L$, lung; DA, descending aorta; MP, left mediastinal pleura; $P$, pericardium; blue arrow, thoracic duct. just over the fascia, which appears like a curtain on a wall (Figs. 5B, 6B). Once the nerve is identified, the lymphoadipose tissue in the medial part is sharply dissected out from the nerve. At this point, the esophageal branches of the left RLN should not be cut to maintain esophageal suspension (Fig. 5C). All lymphatic tissues within the visceral fascia are carefully removed (Fig. 5D). Then, the esophageal branches of the left RLN are cut with scissors (Fig. 5E), and the visceral fascia between the nerve and the esophagus is cut to fully mobilize the esophagus. Lymph nodes at the tracheobronchial angle and the infra-aortic area are dis- sected completely until the left pulmonary artery is identifiable (Fig. 5F).

\section{Middle to lower mediastinum dissection}

The dorsal side of the mediastinal pleura is opened along the ventral margin of the azygous vein. The next step is to dissect the duct (Fig. 7A) and lymphoadipose tissue along the ventral border of the descending aorta. The esophagus is retracted toward the ventral side and dissected along the 
ventral margin of the descending aorta until the left mediastinal pleura (Fig. 7B). The left mediastinal pleural and lymphoadipose tissues of the left pulmonary ligament are dissected en bloc with the esophagus. Subsequently, all lymphoadipose tissue adjacent to the esophagus is dissected from the pericardium (Fig. 7C). The esophagus is pulled up to the right dorsal side. The right mediastinal pleural and lymphoadipose tissues of the right pulmonary ligament are concurrently dissected with the esophagus (Fig. 7D). After the subcarinal lymph nodes and both hilar lymph nodes are detached, the esophagus is completely mobilized. A chest tube is inserted into the right pleural cavity.

\section{Bilateral selective neck dissection}

The patient is repositioned in the supine position. A soft gel roll is placed at the level of the acromion process to aid neck extension. At our institution, a collar incision is performed by transverse incision within a skin crease of approximately $2 \mathrm{~cm}$ or 2 fingerbreadths above the superior edge of the clavicle and sternal notch in a curvilinear fashion. The supraclavicular lymph nodes (Fig. 8A) and cervical paraesophageal lymph nodes (Fig. 8B) are routinely dissected in all patients (Fig. 8C).

\section{Gastric mobilization and anastomosis}

For gastric mobilization, robot-assisted laparoscopy or open laparotomy is performed according to the patient's physical characteristics and preferences. At our institution, both paracardial lymph nodes, lesser curvature lymph nodes, lymph nodes along the left gastric artery, lymph nodes along the common hepatic artery, lymph nodes along the celiac artery, and lymph nodes along the proximal splenic artery are routinely dissected. In most patients, a narrow gastric tube is created along the greater curvature of the stomach using linear staplers, and the staple line is then covered with seromuscular sutures. Pyloroplasty is not performed because we use a narrow gastric tube. The gastric tube is wrapped with a vinyl sheet and pulled up to the neck via a retrosternal route.

At our institution, cervical hand-sewn, double-layered end-to-side esophagogastrostomy is routine. For this procedure, the esophageal wall is divided into a muscular layer and a submucosal layer. The anterior wall of the gastric tube is incised. Interrupted suturing of the muscular layer of the esophagus and the seromuscular layer of the gastric tube is performed, and continuous suturing of the mucosal layers is performed from the posterior side to the anterior side.

\section{Discussion}

McKeown esophagectomy with cervical esophagogastrostomy is the standard surgical approach at our institution. The traditional McKeown approach includes thoracic esophageal dissection via right thoracotomy, gastric mobilization via laparotomy, cervical lymph node dissection, and cervical anastomosis [14]. There are several advantages of the McKeown approach over the Ivor Lewis approach; the former (1) allows more comprehensive lymph node dissection and (2) avoids morbidities associated with intrathoracic anastomosis leakage [15]. However, thoracotomy can lead to increased pulmonary complications, especially in patients with poor pulmonary function. With the goal of
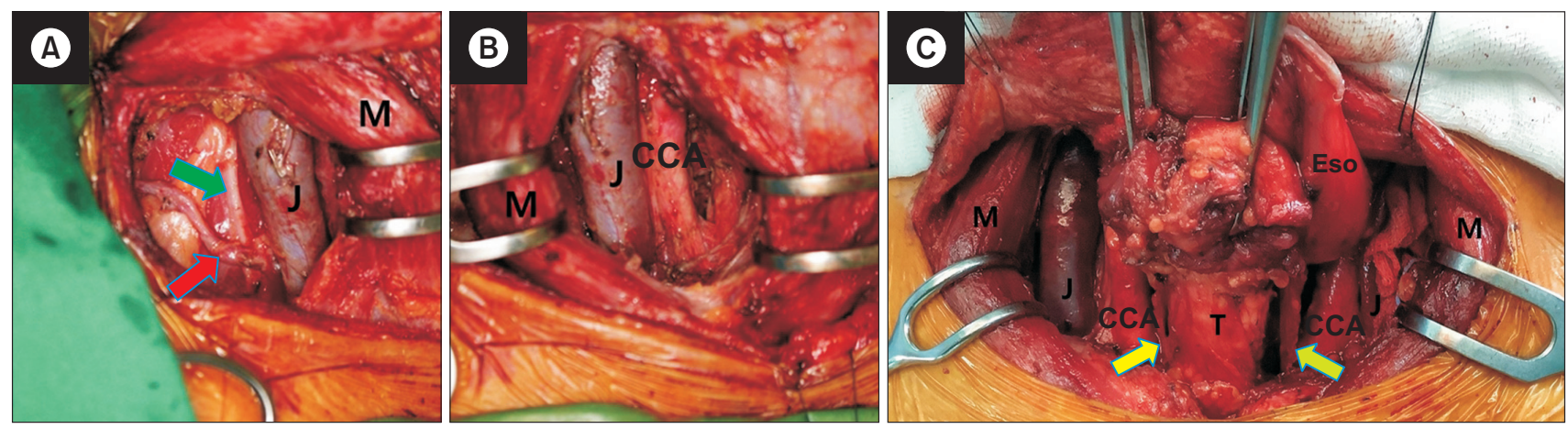

Fig. 8. Bilateral selective neck dissection. (A) Post-dissection of supraclavicular lymph nodes. (B) Post-dissection of cervical paraesophageal lymph nodes. (C) Post-bilateral selective neck dissection. J, jugular vein; $M$, sternocleidomastoid; CCA, common carotid artery; Eso, esophagus; T, trachea; red arrow, transverse cervical artery; green arrow, phrenic nerve; fluorescent yellow arrow, right recurrent laryngeal nerve; fluorescent green arrow, left recurrent laryngeal nerve. 
decreasing complication rates from such an invasive procedure, various minimally invasive approaches have been developed, including a robot-assisted approach. The advantageous robotic surgery platform provides superior visualization with 3-dimensional vision, more degrees of freedom with the endo-wrist function, and more precise motion with tremor filtering and motion scaling.

It is challenging to completely clear all lymph nodes around the RLN because traction, compression, or thermal injury at any single point leads to nerve palsy. Robot-assisted thoracoscopic surgery has been reported to help reduce these complications. We have previously reported that robot-assisted thoracoscopic surgery has favorable outcomes in terms of left RLN lymph node chain dissections [6,7]. In a prospective study by Suda et al. [16], robot-assisted thoracoscopic surgery significantly reduced the incidence of vocal cord palsy, hoarseness, and time on the ventilator.

In addition to the application of robot-assisted thoracoscopic surgery, the fascial plane dissection technique helps reduce surgical morbidity. It is based on a specific anatomic concept regarding the relationship between lymphatic structures and the esophagus. This concept is crucial for minimizing the risk of nerve injury during radical lymph node dissection along the RLN. In general, multiple factors affect the occurrence of RLN palsy, including (1) anatomical factors (e.g., the diameter of the nerve and vascular anomaly), (2) patient factors (e.g., a T3 or deeper tumor in the retrotracheal area, a bulky metastatic node along the RLN, a previous history of surgery or irradiation, or high body mass index), and (3) procedural factors (e.g., traction, compression, or thermal injuries to the RLN during dissection). Applying the fascial plane dissection technique in RAMIE eliminates procedural factors, reducing the incidence of RLN palsy to $<10 \%$.

In conclusion, the fascial plane dissection technique based on microanatomy could be a reliable method for radical esophagectomy using RAMIE.

\section{Conflict of interest}

No potential conflict of interest relevant to this article was reported.

\section{ORCID}

Byung Jo Park: https://orcid.org/0000-0001-5217-4764

Dae Joon Kim: https://orcid.org/0000-0002-2182-010X

\section{References}

1. Van Hillegersberg R, Boone J, Draaisma WA, Broeders IA, Giezeman MJ, Borel Rinkes IH. First experience with robot-assisted thoracoscopic esophagolymphadenectomy for esophageal cancer. Surg Endosc 2006;20:1435-9.

2. Galvani CA, Gorodner MV, Moser F, et al. Robotically assisted laparoscopic transhiatal esophagectomy. Surg Endosc 2008;22:188-95.

3. Espat NJ, Jacobsen G, Horgan S, Donahue P. Minimally invasive treatment of esophageal cancer: laparoscopic staging to robotic esophagectomy. Cancer J 2005;11:10-7.

4. Weksler B, Sharma P, Moudgill N, Chojnacki KA, Rosato EL. Robot-assisted minimally invasive esophagectomy is equivalent to thoracoscopic minimally invasive esophagectomy. Dis Esophagus 2012;25:403-9.

5. Kim DJ, Park SY, Lee S, Kim HI, Hyung WJ. Feasibility of a robot-assisted thoracoscopic lymphadenectomy along the recurrent laryngeal nerves in radical esophagectomy for esophageal squamous carcinoma. Surg Endosc 2014;28:1866-73.

6. Park SY, Kim DJ, Yu WS, Jung HS. Robot-assisted thoracoscopic esophagectomy with extensive mediastinal lymphadenectomy: experience with 114 consecutive patients with intrathoracic esophageal cancer. Dis Esophagus 2016;29:326-32.

7. Park SY, Kim DJ, Kang DR, Haam SJ. Learning curve for robotic esophagectomy and dissection of bilateral recurrent laryngeal nerve nodes for esophageal cancer. Dis Esophagus 2017;30:1-9.

8. Park SY, Kim DJ, Do YW, Suh J, Lee S. The oncologic outcome of esophageal squamous cell carcinoma patients after robot-assisted thoracoscopic esophagectomy with total mediastinal lymphadenectomy. Ann Thorac Surg 2017;103:1151-7.

9. Kim DJ, Park SY, Hong MH. Korean experiences of the treatment of esophageal squamous cell carcinoma. In: Ando N, editor. Esophageal squamous cell carcinoma: diagnosis and treatment. 2nd ed. Singapore: Springer Nature Singapore Pte Ltd.; 2020. p. 363-76.

10. Ding X, Zhang J, Li B, et al. A meta-analysis of lymph node metastasis rate for patients with thoracic oesophageal cancer and its implication in delineation of clinical target volume for radiation therapy. Br J Radiol 2012;85:e1110-9.

11. Wang Y, Zhu L, Xia W, Wang F. Anatomy of lymphatic drainage of the esophagus and lymph node metastasis of thoracic esophageal cancer. Cancer Manag Res 2018;10:6295-303.

12. Tachimori Y, Nagai Y, Kanamori N, Hokamura N, Igaki H. Pattern of lymph node metastases of esophageal squamous cell carcinoma based on the anatomical lymphatic drainage system. Dis Esophagus 2011;24:33-8.

13. Park SY, Suh JW, Kim DJ, et al. Near-infrared lymphatic mapping of the recurrent laryngeal nerve nodes in T1 esophageal cancer. Ann Thorac Surg 2018;105:1613-20.

14. McKeown KC. Trends in oesophageal resection for carcinoma with 
special reference to total oesophagectomy. Ann R Coll Surg Engl 1972;51:213-39.

15. D’Amico TA. Mckeown esophagogastrectomy. J Thorac Dis 2014;6(Suppl 3):S322-4.
16. Suda K, Ishida Y, Kawamura Y, et al. Robot-assisted thoracoscopic lymphadenectomy along the left recurrent laryngeal nerve for esophageal squamous cell carcinoma in the prone position: technical report and short-term outcomes. World J Surg 2012;36:1608-16. 